\title{
Gianni Celati, lo spazio d'assenza e Gianni Celati, lo spazio d'assenza e il negativo del visibile
}

\section{Matteo Martelli}

\section{(2) OpenEdition}

1 Journals

Edizione digitale

URL: https://journals.openedition.org/cher/668

DOI: $10.4000 /$ cher.668

ISSN: 2803-5992

\section{Editore}

Presses universitaires de Strasbourg

\section{Edizione cartacea}

Data di pubblicazione: 9 juillet 2020

Paginazione: 33-44

ISBN: 979-10-344-0068-3

ISSN: 1968-035X

\section{Notizia bibliografica digitale}

Matteo Martelli, «Gianni Celati, lo spazio d'assenza e Gianni Celati, lo spazio d'assenza e il negativo del visibile», reCHERches [Online], 24 | 2020, online dal 20 septembre 2021, consultato il 21 novembre 2021. URL: http://journals.openedition.org/cher/668 ; DOI: https://doi.org/10.4000/cher.668 


\section{Gianni Celati, lo spazio d'assenza e il negativo del visibile}

Matteo Martelli ${ }^{1}$

$\mathrm{I}_{\mathrm{t}}^{\mathrm{n}}$ n questo contributo vorrei riprendere e rileggere le pagine finali di uno dei testi più suggestivi che Gianni Celati ha dedicato ai temi della percezione, dello sguardo e delle maniere in cui l'universo visivo è stato pensato e tradotto nel tempo, in opere letterarie, artistiche o filosofiche. Il testo a cui mi riferisco è "Collezione di spazi», saggio nato in occasione di un intervento all'Università di Bologna, nel 2002, e successivamente pubblicato sulla rivista il verri. In quelle pagine, come è noto, Celati traccia alcune linee di un'archeologia dello sguardo moderno, in un intenso e appassionato passaggio tra il "qui si vede questo » e il «qui qualcuno sta vedendo qualcosa» (Celati 2003: 61 e 65), ossia nel passaggio tra forme di descrizione date da un criterio d'ordine in cui «non esiste l'incertezza della percezione» a quello che potremmo chiamare un sentirsi nella (e della) percezione. L'assunzione di base che guida l'argomentazione dello scrittore è data nelle prime righe: «Si descrivono spazi per capire che cosa percepiamo là fuori, come pensiamo e come ci figuriamo questa cosa avvolgente in cui siamo piazzati, che non ha sostanza perché sembra un puro vuoto tra i corpi, e che però è sempre anche una modalità d'affezione» (Celati 2003: 57-58).

Il gesto descrittivo, ma anche il suo risultato artistico in quanto quadro, testo o fotografia, è dunque per Celati un momento di riflessione teso a interrogare una relazione puntuale, puntiforme, con l'esterno, con quanto, con l'autore, può dirsi il sentimento dello spazio. È questa del resto l'espressione che dà il titolo a un'intervista del 1991 durante la quale Celati aveva dato una bella definizione del suo rapporto al mondo visuale. Le immagini, affermava in quell'occasione Celati, sono "apparenze, cioè fenomeni di luce», e « $[\mathrm{t}]$ utto quello che possiamo chiedere alle immagini è di lasciare le cose e le persone nel loro spazio, di farci sentire la loro distanza» (Celati 1991: 27). Apparenze, si sa, è uno dei termini

1 Matteo Martelli, Université de Strasbourg, CHER. 
chiave di Celati, qui riferito al mondo iconico, alla fotografia in particolare, ma altrove all'esterno, a uno spazio osservabile forse proprio in quanto può rispondere, bene o male, euforicamente o disforicamente, al gesto di lettura dell'osservatore. Le apparenze sono il mostrarsi delle cose, il loro offrirsi come fenomeno (Celati 2003: 63). Tutto il testo "Collezione di spazi» ruota dunque intorno a questo mostrarsi, a uno studio delle apparenze di fronte alle quali un soggetto si trova costantemente immerso, avvolto, intricato: sorpreso e sospeso in un ritmo, entro "la risonanza di una percezione visiva» (Celati 2003: 78).

Qualche anno fa, Marco Sironi aveva dato una giusta definizione del legame che nell'opera di Gianni Celati sussiste tra l'attività percettiva e la scrittura, sottolineando come quest'ultima fosse un costante "esercizio di sguardo e di ascolto [che] implica sempre un atto di lettura» (Sironi 1994: 91), ossia una forma di partecipazione e comprensione dell'esterno. "In questo senso", continuava Sironi, scrivere "è qualcosa come un "lasciarsi scrivere" - dagli eventi, dal momento presente, dalle cose come appaiono qui, adesso » (Sironi 1994: 91). Ciò che accomuna scrittura e osservazione è dunque un'esposizione, il portarsi verso il fuori, in ascolto, in visione. Al centro della questione posta se ne trova difatti una seconda: quella dell'abitabilità del sensibile, ossia quella maniera propria del sensibile di pensarsi in me (Merleau-Ponty 1945: 248). La cosa avvolgente pensa (pesa, direbbe Jean-Luc Nancy), in me; essa quindi «è prima di tutto un'affezione: un effetto che disturba l'uniformità dello spazio; e come tutte le affezioni a cui non si resiste porta disordine, anche là dove il fuori è tutto regolato da un principio d'ordine» (Celati 2003: 63).

Celati pensa in questo caso all'atto percettivo secondo un motivo particolare, quello dell'intermittenza. Si tratta di una lettura che all'interno del visivo, in quello spazio discontinuo che è il visivo (ossia entro lo spazio delle modificazioni di un visibile ininterrotto), rende conto di un campo attenzionale che si compone di intensità, intrusioni e ritmi che emergono secondo dei phrasés dello sguardo, tali che la visione, scrive Celati recuperando Merleau-Ponty, è ogni volta " "un ordine nascente" [che] non si presenta mai come dato, [ma] è sempre un fenomeno vibratorio in atto nel presente» (Celati 2003: 83). Ė in tal senso che tale intermittenza, o quella "variabilità che è lo sguardo", fa dell'atto stesso del vedere « una ricerca per "vedere", [...] un'inquietudine per capire cosa vediamo nella mente» (Celati 2008b: 128). Chiedere a un'immagine di farci sentire le cose nel loro spazio è in fondo domandare un posizionamento, una maniera d'osservare e d'orientarsi davanti a quanto emerge: di fronte a quanto, come apparenza, ha natura sospesa. Al tempo stesso, ciò significa interrogare, insieme all'immagine o allo spazio, lo sguardo dell'osservatore, la postura di colui che partecipa all'esperienza di visione, secondo uno sguardo che impegni, insieme alla cosa vista, la materialità del corpo e la vibrazione affettiva di colui che sente e di ciò che è sentito, come parti inseparabili di quanto entra nel campo della percezione. Il sentimento di distanza, che lo scrittore chiama spesso una misura (come relazione, inquadramento e avvolgimento insieme), può essere in tal senso 
considerato come una cerniera sensibile dell'esterno, cioè della nostra maniera di accogliere quest'ultimo, di esserne toccati e di rispondervi.

E all'interno di questo quadro che le ultime pagine di «Collezione di spazi» affrontano un'esperienza di visione perturbante ed enigmatica, che riprende e al tempo stesso prolunga il percorso celatiano, mettendo in evidenza l'eredità merleau-pontiana della sua riflessione. In quegli ultimi paragrafi difatti l'attenzione dello scrittore si sposta sul lavoro di due artisti, Alberto Giacometti ${ }^{2}$ e Rachel Whiteread (ai quali si aggiungono dei brevi passaggi sulla pièce di Samuel Beckett, Not I). Ciò che conduce all'accostamento di questi autori è ancora una volta il motivo della distanza, ma la sua esplorazione passa ora attraverso una forma plastica, presente anche in Not I, quella dell'impronta. Il tema dominante del saggio si pone in tal modo di fronte a una particolare forma di visione, quella delle assenze, delle lacune, a una visione che lavora su un ritiro, una perdita e una sopravvivenza insieme, e che fa di questo stesso movimento la materia, le pointillage, del visuale. Una maniera d'accogliere, nella stessa percezione, la materialità di un'assenza, di aprire la visione su un negativo del visibile. Un'impronta, ossia il segno lasciato da un corpo su un altro.

Nelle prime righe de «Le doute de Cézanne», Merleau-Ponty scrive: «ce que nous appelons son œuvre n'était pour lui que l'essai et l'approche de sa peinture» (Merleau-Ponty 1996: 13). Le parole del filosofo francese, il cui saggio - insieme a L'Eil et l'Esprit - è citato più volte in "Collezione di spazi », offrono una prima occasione per delineare il dialogo celatiano con l'opera di Alberto Giacometti (un dialogo, bisogna ricordarlo, di lunga durata, ma al tempo stesso sovente carsico). Con i termini essai e approche, Merleau-Ponty vuole difatti sottolineare una precisa linea di ricerca presente nel lavoro di Cézanne che tocca in primo luogo alcune modalità del nostro rapporto al mondo. In Cézanne, ciò a cui assistiamo è un ritorno alle cose in quanto cose, a un ordine nascente della materia, a un mondo primordiale (Merleau-Ponty 1996: 18), ossia a un'adesione percettiva al mondo (Carbone 2001: 21). Essai e approche definiscono in tal modo tanto una forma di contatto ed esposizione tra soggetto e spazio esterno quanto la loro manifestazione pittorica, l'espressione di una percezione vissuta in cui «la vision manifeste [des choses] se double en lui [dans mon corps] d'une visibilité secrète» (Merleau-Ponty 1964: 22).

Si toccano qui alcuni punti chiave attraverso cui individuare lo sforzo espressivo di Cézanne, le doute, atto di creazione e maniera d'essere insieme, per cui nei suoi dipinti, scrive Merleau-Ponty, si rende presente lo spettacolo (ossia le apparenze) di cui gli uomini fanno parte senza vederlo. Il punto su cui insiste

2 I paragrafi dedicati a Giacometti in "Collezione di spazi» si ritrovano, rielaborati e con alcune varianti, anche in altre pubblicazioni di Celati: «Giacometti, la percezione e il dentro dello spazio » (Celati 2008a) e " Giacometti e il dentro dello spazio » (Celati 2011c). 
il filosofo è questo: Cézanne «reprend et convertit justement en objet visible [...] la vibration des apparences qui est le berceau des choses» (Merleau-Ponty 1996: 23). Attraverso l'opera quanto si espone come oggetto visibile è dunque una latenza, che il quadro stesso ci offre allo sguardo, che lì affiora ed emerge. È questo, inoltre, il senso che si può dare a un noto passaggio presente ne L'Eil et l'Esprit che ha molteplici implicazioni per il nostro percorso: «je serais bien en peine de dire où est le tableau que je regarde. Car je ne le regarde pas comme on regarde une chose, je ne le fixe pas en son lieu, [...] je vois selon ou avec lui plutôt que je ne le vois» (Merleau-Ponty 1964: 23). Guardare selon ou avec è sentire questo inesplorato - questo impensato -, percepire un luogo che prende forma entro la presenza singolare di un'invisibilità che si manifesta (cfr. Carbone 2011: 11-13).

Qualcosa di simile si esprime nelle pagine che Celati dedica all'opera e al lavoro di Alberto Giacometti. Se da un lato queste portano a conclusione un lungo e denso percorso sullo spazio e la sua rappresentazione, dall'altro esse circoscrivono la costante e quasi infinita ricerca di una forma da parte dello scultore, la sua maniera di vedere e di ritagliare la superficie sensibile entro un lavoro che pone dei rilievi e degli accenti che costituiscono anzitutto una disposizione di fronte dell'esperienza. In quelle pagine si può dunque vedere tanto un omaggio a una figura-guida (Belpoliti e Sironi 2008: 63 e 114), quanto un'interrogazione su un'opera che permette a Celati di mobilitare nel suo testo una propria riflessione sulle arti visive e sul visibile. Alla base di questo dialogo vi è ancora una questione d'essai e d'approche, e di quanto essi sottendono nella dinamica dell'operare dell'artista, come indica chiaramente Giacometti in un'intervista ad Antonio Del Guercio nel 1962:

Me ne infischio del problema di fare un bel quadro, o di finirlo. Vedo la persona che mi sta davanti come una cosa complessa, contraddittoria: perciò, per comprenderla, devo copiarla [...]. Le mie pitture sono copie non riuscite della realtà. [...] Il giorno che si arrivasse a capire totalmente una certa cosa, la si potrebbe rifare. Ma ciò è impossibile, poiché quella cosa e io siamo dentro il movimento e il mutamento continuo della vita, che non può essere immobilizzato. (Giacometti 2013: 256-257)

Questa «sua strana vicenda», come la chiama Celati, che prende avvio nella metà degli anni Trenta, è il ritorno alla pratica della copia dal vero, ossia la volontà dello scultore di rappresentare una testa come la si vede che si scontra, nell'ottica di Giacometti, con «l'impossibilità effettiva» della sua realizzazione. Agli inizi degli anni Sessanta, in un'intervista per la televisione svizzera, lo scultore ritorna su quel passaggio fondamentale nel suo percorso artistico. Ciò che importa, afferma lo scultore, è "provare», anche se "non ho ancora capito il perché [...] sia quasi impossibile» (Soavi 2000: 45). Il termine centrale, come per Cézanne, è appunto provare, tanto nel senso di cimentarsi, senza sosta, a dare forma e modellare quanto dimora come un mistero, quanto nel senso di aderire costantemente a un sentimento, a un'emozione, che nasce di fronte al modello, alla sua visione, alla cosa «complessa [e] contraddittoria». Lorizzonte 
e limite di questo lavoro, entro il quale si gioca la sfida espressiva di Giacometti, è quello della somiglianza, parola ambigua attraverso la quale l'artista esprime unopposizione tra una coppia di termini: quelli di idea e concetto, da un lato; e quelli di visione e percezione dall'altro. Provare è anzitutto sentire e mettere alla prova la somiglianza, interrogarla, sondarla, toccarla, scostandosi da un'idea della scultura come rappresentazione di un concetto - " un oggetto, se lo penso, è una cosa che sapevo" (Soavi 2000: 44) - per realizzare la propria visione.

Da questo punto di vista, la visione giacomettiana si esprime in primo luogo come un accesso al mondo, legandosi profondamente all'indagine celatiana sui modi di presentarsi del sensibile che il soggetto incontra entro un'oscillazione costante «tra l'attenzione e lo smarrimento» (Sironi 2004: 45). Siamo qui all'interno di quel piano estetico che Celati rivendica come accoglienza dei modi del «sensitivo", una maniera di pensare l'esterno secondo un'aderenza, una " coniugazione con quello che è fuori di noi, [...] qualcosa che è un momento che poi scappa via» (Celati 2008c: s.p.). Tra i testi di Giacometti, c’è allora un passaggio chiave che Celati cita e a partire dal quale sviluppa la sua riflessione.

On peut s'imaginer que le réalisme consiste à copier... un verre tel qu'il est sur la table. En fait, on ne copie jamais que la vision qu'il en reste à chaque instant, l'image qui devient conscience... Vous ne copiez jamais le verre sur la table; vous copiez le résidu d'une vision. J'ai compris peu à peu la réalité d'un certain phénomène quon appelle la sculpture. Lorsque je regarde le verre, de sa couleur, de sa forme, de sa lumière, il ne me parvient à chaque regard qu'une toute petite chose très difficile à déterminer, qui peut se traduire par un tout petit trait, par une petite tache, chaque fois que je regarde le verre, il a l'air de se refaire, c'està-dire que sa réalité devient douteuse, parce que sa projection dans mon cerveau est douteuse, ou partielle. On le voit comme s'il disparaissait... resurgissait... disparaissait... resurgissait... c'est-à-dire qu'il se trouve bel et bien toujours entre lêtre et le non-être. (Giacometti 2013: 245)

Ciò che si copia non è che il residuo di una visione. È questa l'espressione centrale per Gianni Celati. Nell'orizzonte dello scrittore, la postura di Giacometti assume un duplice valore. Da un lato essa implica uno smarrimento, una perdita, ossia "il crollo di tutto ciò che si è chiamato rappresentazione del mondo " (Celati 2008a: 118). Il gesto di Giacometti è quello di chi si sottrae a una tradizione d'immagini (a uno schermo di immagini, afferma l'artista) per ritrovarsi di fronte a una realtà molto più misteriosa e perturbante. D'altro lato, l'operato artistico che ne emerge diviene il campo, continuo e senza fine, per interrogare quello smarrimento, per interrogare la propria visione: «une sculpture ne m'intéresse vraiment que dans la mesure où elle est, pour moi, le moyen de rendre la vision que j'ai du monde extérieur... Ou, plus encore, elle n'est aujourd'hui pour moi que le moyen de connaître cette vision» (Giacometti 2013: 178). La questione della copia diventa così per lo scrittore un'apertura verso il come dell'esperienza visiva.

C'è un'espressione curiosa, che Celati recupera nel suo testo, con cui Giacometti definiva quest'esperienza: l'espressione è «apparition incertaine» (Giacometti 2013: 578). Ciò che vediamo non è altro che un apparire, 
incerto e instabile, poiché è preso entro un movimento continuo, entro una continua vibrazione (quanto, ancora con Merleau-Ponty, potremmo chiamare la palpitazione dello sguardo). Il come della visione si lega a un ritmo, a una sospensione in movimento delle cose e degli spazi, per la quale «les têtes, les personnages ne sont que mouvement continuel du dedans, du dehors, ils se refont sans arrêt»(Giacometti 2013: 578). Apparizione incerta, dunque, ma anche ciò che è possibile ancora chiamare, evocare, modellare. Nell'incessante operare di Giacometti, è noto come durante gli anni Quaranta le statue tendessero a rimpicciolirsi sotto le sue mani, in una taglia che poteva ridursi fino a pochi centimetri, fino a sparire. Questa riduzione ultima delle sculture dell'artista, questo "passage à la limite» (Bonnefoy 2012: 277), ha ancora certo a che fare con la ricerca della somiglianza: il tentativo di (ri)fare una testa, quella testa nella sua "finitezza», "amplificare e mettere in salvo l'ora riflessa del tempo », ciò che pone, o impone, anche la possibilità della sua «sparizione" (Barone 1999: 48).

Apparizioni incerte, allora, che possono sparire, in un lavoro che in questa ricerca, scrive Celati, «diventa la pratica d'una sintomatologia, per esplorare una dimensione che possiamo chiamare l'inconscio quotidiano» (Celati 2008a: 118). L'espressione che usa Celati, sintomatologia, traduce una relazione precisa di questa caducità o sospensione delle opere, del loro possibile non-esserci. Essa non soltanto si oppone a un sistema simbolico, in riferimento anzitutto alle sculture-oggetto di Giacometti nel suo periodo surrealista (cfr. Dupin 1991: 39), ma richiama l'attenzione celatiana, di lunga durata, per il tema della traccia, per « un insieme di emergenze sintomatiche che ci mostrano qualcosa in lontananza senza delineare i contorni» (Palmieri 2017: 429). Il motivo dell'apparizione si congiunge in tal modo a quello del residuo della visione, a ciò che si ritira e che ritirandosi ancora resta: non già la testa del modello o il bicchiere sul tavolo, ma ciò che è rimasto, "un'assenza in una presenza» (Celati 2003: 89). Assenza, qui, non significa però vuoto, perdita, ma ritiro, distanza, allontanamento, spostamento di ciò che si mostra da una lontananza. L'assenza non è ciò che è scomparso, ma ciò che è tale in una relazione di distanza, ciò che se ne va in ogni venuta in presenza (cfr. Nancy 2001: 155-157). Il discorso di Celati apre qui un ulteriore elemento della sua riflessione sul visibile, poiché in questo apparire incerto, nel sorgere di ciò che si ritrae, quanto è dell'ordine della perdita sembra paradossalmente farsi luogo, il luogo stesso della visione: «place vide de l'absente comme place non vide [...]: place d'un déplacement» (Nancy 2003: 128).

Nell'edizione del saggio pubblicata sulla rivista Riga, Gianni Celati ricorda una grande mostra delle opere di Giacometti tenutasi a Parigi (Celati 2008a: 119). È questa l'occasione per spostare il discorso sulla visione del lavoro dello scultore, ossia per vedere in esso l'apertura di un'ulteriore collezione di spazi, attraverso lo stare davanti delle statue. $\dot{\mathrm{E}}$ in fondo questo il dato esperienziale che muove il discorso e il pensiero visuale di Celati: uno stare davanti dell'opera, 
di ciò che «se tient devant: ni ici ni ailleurs, ni présent ni absent, mais bien imminent» (Alloa 2011: 18).

L'idea di Celati è che le sculture dell'artista svizzero non soltanto si trovino entro uno spazio, ma esse stesse si facciano spazio, o meglio si facciano luogo di una visione: "quello che Giacometti ha in mente è che, per non essere un concetto convenzionale [...], la scultura debba essere un punto dove sorge una visione, con un suo interno" (Celati 2003: 89). Per comprendere questo farsi luogo della scultura, è possibile soffermarsi su una nozione apparentemente banale sulla quale Celati termina le sue pagine dedicate a Giacometti. Le sculture dell'artista svizzero, sottolinea lo scrittore, sono nella maggior parte calchi, fusioni in bronzo o calchi di gesso di precedenti opere in positivo composte di varie terre. È il calco a interrogare quindi Celati:

Quelle superfici sono solo materia, come un residuato metallico, materia compressa verso il suo asse centrale, da cui sembra per caso sia saltata fuori una figurina allungata con tratti molto sommari. [...] Tutto è inerte e in riposo, un'assenza in una presenza. In quel punto vedo solo una figurina che mi colpisce per come è inconcepibilmente magra e allungata. Diciamolo così: tutte le vedute di quelle statue che accumulo ispezionandole da una parte e dall'altra, sono il negativo di un interno di quello spazio verso cui sono attratto. (Celati 2003: 89)

L'esperienza di cui parla Celati sembra avere qualcosa di singolare. Lo stesso scrittore, di fronte alla superficie "rugosa, irregolare, sbalzata» delle statue, avverte una sorta di smarrimento. Il suo movimento, il girare attorno, si apre con un "non so cosa vogliano dire» (Celati 2003: 89), ed è soltanto quando lo sguardo dello scrittore si porta verso la materialità dell'opera, il gesto di «implosione» di cui ora vede il negativo, che ne percepisce la visione. A partire da questa esperienza, ulteriore variazione di «tecniche di oscillazione e sospensione» (Spunta 2008: 161) dello sguardo, girando da una parte all'altra delle statue, conclude:

Ora le sue statue mi appaiono per quel che sono: sono dei calchi in bronzo, il negativo degli stampi, in cui sè depositata la forma di una visione; una visione che nasceva nel momento in cui delle mani manipolavano l'argilla, scavandola e comprimendola sempre di più verso un suo interno invisibile. Argilla che poi è stata messa in uno stampo, di cui ora vedo l'inverso, nella forma di materia rugosa che si presenta ai miei occhi. Lo spazio esterno di quelle superfici è il negativo d'una visione, ora in riposo nel suo spazio. (Celati 2003: 90)

Ciò che colpisce Celati è il passaggio tra l'argilla lavorata da Giacometti e lo stampo, l'impronta che essa ha lasciato nel gesso per poter in seguito ottenere l'anima (il nuovo positivo) che sarà la base del calco in bronzo. Quel passaggio, ci dice lo scrittore, è l'impronta di una visione. Visione che è ora al di sotto della superficie visibile, depositata entro un salto di materia, entro un contatto che ne ha visto l'allontanamento, il formarsi di una distanza ora visibile.

La forma dell'impronta alla quale si riferisce Celati, non diversamente dalla somiglianza, si muove entro un'ambiguità. Geroges Didi-Huberman, che ha dedicato numerose pagine a questa nozione e alle sue implicazioni visuali e 
artistiche (Didi-Huberman 2001 e 2008), ricorda come l'impronta ci ponga anzitutto di fronte a una dialettica del visivo, facendo riemergere nel visibile la questione del tangibile (Didi-Huberman 2008: 191). L'ambiguità è qui quella di un pensiero che si pone davanti a una forma di contatto paradossale, di quanto è presente senza restare, di quanto, allontanandosi e ritirandosi, per quel suo stesso movimento si offre alla visione. Nel gesto dell'impronta, ciò con cui ci confrontiamo è lo scarto, l'apertura, di ciò che genera con la sua stessa forma - con l'impressione - un'invisibilità tattile. I calchi di Giacometti si fanno così sopravvivenza materiale, visibile, di una pratica e un'esperienza: nel passaggio da una materia a un'altra, da un'assenza a una presenza, le opere mostrano in ciò che resta, nel loro negativo, il movimento della visione entro il quale permane un atto di attraversamento, una incitazione che si ripete. Figure dell'ambiguità, soglie, esse sono una costellazione "che anima uno spazio, una visione che riposa dentro punti dello spazio, all'interno dello spazio» (Celati 2003: 91).

Lo stato di riposo della materia, l'inerzia delle statue che attira e interroga Celati, sarebbe dunque questa presenza di una distanza, presenza di un movimento, della materia compressa «sempre di più verso il suo interno invisibile». Seguendo questa linea di pensiero Celati individua infine nelle opere di Giacometti "la scoperta di un dentro dello spazio", di una visione che nasce dal negativo della materia, in quanto forma di pensiero figurale. Di fronte a quelle figure filiformi, al loro "mutismo", la sfida celatiana sembrerebbe essere quella di lasciare la materia sollevarsi, aprirsi, dare luogo a quella dialettica visuale che Massimo Rizzante ha definito "un'estetica del reincanto" (Rizzante 1993: 33-34) e che è presente nella sua narrativa (cfr. Spunta 2005: 33-34). Ma la nozione di impronta impegna qui più particolarmente il pensiero di un negativo che si offre allo sguardo, ossia la maniera di accogliere una certa relazione tra visibile e invisibile, che probabilmente Celati ritrova ancora nella riflessione di Merleau-Ponty:

Un certain rapport du visible et de l'invisible où l'invisible n'est pas seulement non-visible (ce qui a été ou sera vu et ne l'est pas, ou ce qui est vu par autre que moi, non par moi), mais où son absence compte au monde (il est «derrière» le visible, visibilité imminente ou éminente [...]) où la lacune qui marque sa place est un des points de passage du «monde». C'est ce négatif qui rend possible le monde vertical $[. .$.$] et le possible comme prétendant à l'existence [. .$.$] et l'«écart»,$ et la totalité par dessus les écarts, - et le rapport pensé-impensé. (Merleau-Ponty 1979: 281)

L'invisibile è un'assenza, certo, ma essa è tale come negativo di un visibile che si presenta, che scava il visibile stesso e che in questo movimento, in questo affioramento in negativo, dà forma a una "concrection de la visibilité». È in questo senso che il discorso che Celati apre osservando la superficie silenziosa delle statue di Giacometti tocca una sua più ampia riflessione sulla visualità. Il sentimento di distanza, di cui si scriveva inizialmente, come cerniera sensibile verso l'esterno trova in questa dialettica una sua manifestazione in cui ciò che 
è possibilità, latenza e chair della cosa (Merleau-Ponty 1979: 175) è accesso al mondo.

Gli ultimi paragrafi di «Collezione di spazi» portano ancora un elemento di riflessione intorno a questa cerniera sensibile. Per parlarne, lo scrittore ci conduce di fronte all'opera plastica di Rachel Whiteread, il cui lavoro procede per calchi di oggetti quotidiani, luoghi di vita ordinaria, spazi abitati in cui "quello che si vede è il negativo della cosa: [...] lo stampo di un vuoto" (Celati 2003: 91).

Lo scrittore si riferisce in questo caso a due opere, Ghost (1990) e House (1993). Nel primo caso, l'osservatore si trova di fronte allo stampo di una stanza, una comune stanza vittoriana il cui calco - ora in un museo - offre al tempo stesso un volume compatto, grigiastro, entro il quale non si può entrare, e la pura esteriorità di una superficie sensibile, la sua pelle (Lawson 2004: 70), segnata in ogni dettaglio da quanto è ora visibile solo come assenza. House è invece il titolo di un progetto più ampio in cui Whiteread ha investito direttamente un luogo, un quartiere del Nord-Est di Londra, attraverso l'impronta in cemento di una casa il cui stampo si è dunque sostituito, per un certo periodo, al modello originale. La natura dei lavori della scultrice rende la loro visione estremamente perturbante, un'inquietudine che coglie lo spettatore di fronte a un'immagine in negativo, a uno spazio che si forma tra le cose e in cui presenza e assenza si mostrano allo stesso tempo, con lo stesso movimento (Tarantino 1996: 98). Lo sguardo percorre l'interno di una forma di perdita, seguendo sulle superfici delle sculture una visione impossibile da circoscrivere («bisogna fare un giro del pensiero per vedere che il materiale [...] è lo stampo di un vuoto», scrive Celati), una visione di quanto è stato in contatto con il gesso o il cemento e che ne ha formato i contorni, le irregolarità, le puntuazioni ritmate, la sua apparenza ora divenuta grisaglia.

Nella lettura delle opere di Whiteread ritornano spesso alcuni termini - perturbante, invisibile, fantasmi (cfr. Whiteread 1995 e 1996, Townsend 2004) - che sono altrettante maniere di porre una domanda che non cessa di sorgere davanti alle sculture. La questione è come potremmo osservare quei calchi senza appoggiare lo sguardo su quanto era in contatto con quelle superfici in gesso, senza vedervi e riconoscervi il movimento di perdita della cosa. Come potremmo avvicinare queste opere senza sentire (provare) la dislocazione figurale di un ritiro, l'allontanamento della cosa nella prossimità della traccia che ci sta davanti. Anche Gianni Celati, riprendendo il titolo di una delle opere, parla di fantasmi: «noi vediamo i fantasmi delle cose. Non si sa come guardarli. Sono cose che appaiono nella loro scomparsa» (Celati 2003: 91). Non si tratta di fantasmi di un passato, o di una sua nostalgia, precisa lo scrittore, bensì di ombre che si mostrano nella loro possibilità d'essere, nel luogo della loro scomparsa. Lo stampo, il negativo del visibile, tocca un vuoto, il «fantasma di una forma di vita» che per un certo periodo ha offerto allo sguardo degli osservatori 
l'inquieto e «commovente» spettacolo della visione di « uno spazio d'assenza e d'affezione» (Celati 2003: 91).

Il tema del fantasma, che chiude l'articolo di Celati, ci riporta in tal modo verso un duplice interrogativo che lo scrittore si pone in queste pagine, quello della forma e della presenza da un lato, e quello della latenza dall'altro, ossia il come del darsi delle cose nello spazio, di fronte a un sguardo che osserva con o secondo l'opera guardata. Quella sorta di cubo di gesso che è Ghost, così appena segnato, dentellato da una materia svanita che ora ne disegna la superficie esteriore è presenza immanente, ma di una distanza. Essa ci obbliga a pensare a questa presenza come al processo di ciò che svanisce (Didi-Huberman 1992: 84). Figura d'ombra, il fantasma è il diafano dell'apparizione di quanto scompare: quella forma di vita che Celati dice «intravista, già sempre dispersa nello spazio, compressa nell'interno del suo spazio» (Celati 2003: 91). Ma, aggiungeva Erwin Straus, la distanza non è sentita, è piuttosto il sentire che rivela la distanza (Straus in Didi-Huberman 1992: 117). È il sentire, allora, secondo la forma, che rivela i fantasmi, ulteriore apparizione incerta, sospesa, perché dischiusura, fenditura sempre in atto nello spazio del visibile.

In un dialogo con Massimo Rizzante, Celati affermava che «le immagini sono uno stato ricettivo a cui ci apriamo » che possiamo cercare di comprendere solo «nel processo con cui ci proiettiamo verso ciò che si configura come un'esperienza e una passione» (Celati 2011b: 73). Ciò che ci tocca dell'immagine, della scultura, e che come passione agita e inquieta il nostro sguardo, è quel luogo d'apparizione di fantasmi, di forme di vita che si dischiudono, in un vedere come adesione al mondo, secondo "quel valore differenziale che è lo sguardo" (Celati 2003: 89), che non smette d'interrogare, e perturbare, il nostro stesso vedere.

L'apparizione incerta di cui parlava Giacometti trova qui una sua declinazione essenziale, quella di uno sguardo che si posa tra l'essere e il non-essere, in cui, nello stare davanti della cosa, quella cerniera sensibile è apertura verso ciò che al tempo stesso ci offre una misura e un orizzonte della visione. L'affezione, la parola guida di Celati, è quest'impressione, quest'impronta sempre presente nelle maniere dello sguardo, questo residuo della visione che ci attraversa, ci coglie e guida il nostro vedere. Osservando le opere di Alberto Giacometti e Rachel Whiteread, Celati in fondo porta la nostra attenzione su ciò che quell'affezione tocca, i fanstami à fleur de peau dell'opera, secondo uno sguardo che fa dell'invisibile la parte saliente dell'opera stessa e della visione, e lo spazio d'assenza ciò che scava ogni visibile. L'impronta è allora la manifestazione figurale di una punteggiatura che Celati stava cercando: quel dentro dello spazio che tocca un esterno, entro la relazione inquieta di una materia (il bronzo, il gesso, il cemento) che dà luogo all'incertezza di una risonanza e all'incitazione di uno sguardo che si muove nel suo distanziamento. Lo spazio d'assenza è accesso sensibile a una visione, a dei mondi di visione, che sono atmosfere, ombre e fantasmi che il negativo del visibile porta, impone e attraverso i quali pensiamo e ci figuriamo quella "cosa avvolgente in cui siamo sempre piazzati». Nelle sue «Notes de travail», Merleau-Ponty scriveva che «la perception est 
non perception de choses d'abord, mais perception des éléments (eau, air...) de rayons $d u$ monde, de choses qui sont des dimensions, qui sont des mondes, je glisse sur ces "éléments" et me voilà dans le monde» (Merleau-Ponty 1979: 271). E in questo scivolare, in questo transito e passaggio, che lo sguardo non solamente tocca, ma è nel mondo, avvolto in ciò che affiorando compone un luogo, entro una distanza "da cui prorompono tutte le estensioni esterne» (Celati 2008a: 119). Distanza, ma anche fessura, crepa sensibile del visibile, all'opera nel visibile, entro il luogo del negativo dell'immagine.

\section{Bibliografia}

Alloa E., 2011, «Entre transparence et opacité - ce que l'image donne à penser», in Penser l'image, a cura di E. Alloa, Dijon, Les Presses du réel, p. 7-23.

Barone P., 1999, Età della polvere. Giacometti, Heidegger, Kant, Hegel, Schopenhauer e lo spazio della caducità, Venezia, Marsilio.

Belpoliti M. e Sironi M. (a cura di), 2008, Gianni Gelati, Riga, 28.

Bonnefoy Y., 2012, Alberto Giacometti. Biographie d'une œuvre, Paris, Flammarion.

Celati G., 1991, «Il sentimento dello spazio», Cinema \& cinema, 18, p. 25-28.

Celati G., 2003, «Collezione di spazi», il verri, 21, p. 57-92.

Celati G., 2008a, «Giacometti, la percezione e il dentro dello spazio », in Belpoliti e Sironi 2008, p. 116-120.

Celati G., 2008b, «Viaggio in Italia con 20 fotografi 20 anni dopo », in Belpoliti e Sironi 2008, p. 126-135.

Celati G., 2008c, «Intervista inedita a Gianni Celati», in Belpoliti e Sironi 2008, s.p., sezione extra, online: www.rigabook.it.

Celati G., 2011a, Conversazioni del vento volatore, Macerata, Quodlibet.

Celati G., 2011b, «Sulla fantasia», in Celati 2011a, p. 70-80.

Celati G., 2011c, «Giacometti e il dentro dello spazio», in Celati 2011a, p. 81-88.

Carbone M., 2001, La visibilité de l'invisible, Hildesheim, Georg Olms Verlag.

Carbone M., 2011, La chair des images: Merleau-Ponty entre peinture et cinéma, Paris, Vrin.

Didi-Huberman G., 1992, Ce que nous voyons, ce qui nous regarde, Paris, Les Éditions de Minuit.

Didi-Huberman G., 2001, Génie du non-lieu. Air, poussière, empreinte, hantise, Paris, Les Éditions de Minuit.

Didi-Huberman G., 2008, La ressemblance par contact. Archéologie, anachronisme et modernité de l'empreinte, Paris, Les Éditions de Minuit.

Dupin J., 1991, Alberto Giacometti, textes pour une approche, Paris, Fourbis.

Giacometti A., 2013, Écrits. Articles, notes et entretiens, Paris, Hermann Éditeur.

Lawson S., 2004, "Sensitive Skin. Infra mince and différance in the Work of Rachel Whiteread», in Townsend 2004, p. 69-84.

Merleau-Ponty M., 1945, Phénoménologie de la perception, Paris, Gallimard. 
Merleau-Ponty M., 1964, L'CEil et l'Esprit, Paris, Gallimard.

Merleau-Ponty M., 1979, Le visible et l'invisible. Suivi de notes de travail, Paris, Gallimard.

Merleau-Ponty M., 1996, "Le doute de Cézanne», in Id., Sens et non-sens, Paris, Gallimard, p. 13-33.

Nancy J.-L., 2001, Les muses, Paris, Galilée.

Nancy J.-L., 2003, Au fond des images, Paris, Galilée.

Palmieri N., 2017, «Il gesto, gli oggetti, le immagini. Gianni Celati nell'animazione del mondo ", in Celati G., Animazioni e incantamenti, con C. Gajani, a cura di N. Palmieri, Roma, l'Orma, p. 421-438.

Rizzante M., 1993, Il geografo e il viaggiatore. Variazioni su I. Calvino e G. Celati, Fossombrone, Metauro.

Sironi M., 1994, Geografie del narrare. Insistenze sui luoghi di Luigi Ghirri e Gianni Celati, Reggio Emilia, Diabasis.

Soavi G., 2000, Alberto Giacometti. Il sogno di una testa, Milano, Mazzotta.

Spunta M., 2005, "Ghirri, Celati e lo "spazio di affezione" », Il lettore di provincia, 124-124, p. 27-39.

Spunta M., 2008, "Verso la foce con Gianni Celati: tra 'antropologia di popolazioni invisibili' e 'estetica del reincanto', in Sinergie narrative. Cinema e letteratura nell'Italia contemporanea, a cura di G. Bonsaver, M. McLaughlin e F. Pellegrini, Firenze, Cesati Editore, p. 153-157.

Tarantino M., 1996, "The Space between Things », in Whiteread 1996, p. 91-99. Townsend C. (a cura di), 2004, The Art of Rachel Whiteread, London, Thames \& Hudson.

Whiteread R., 1995, Home, a cura di J. Lingwwod, London, Phaidon.

Whiteread R., 1996, Shedding Life, a cura di F. Bradley, London, Tate Gallery Publishing. 